\title{
Fatty Acid Composition and Stoichiometry Determine the Angiogenesis Microenvironment
}

Faith Pwaniyibo Samson ${ }^{1}$, Tosin Esther Fabunmi ${ }^{1}$, Ambrose Teru Patrick ${ }^{1}$,

Donghyun Jee ${ }^{2}$, Diana R. Gutsaeva ${ }^{3}$, and Wan Jin Jahng ${ }^{1 *}$

${ }^{1}$ Department of Petroleum Chemistry, American University of Nigeria, Yola, Nigeria

${ }^{2}$ Department of Ophthalmology and Visual Science, St. Vincent's Hospital, College of Medicine, The Catholic University of Korea, Suwon, Korea

${ }^{3}$ Department of Ophthalmology, Augusta University, Augusta, GA, USA

*Correspondence to Wan Jin Jahng, Ph.D.

Email address: wan.jahng@aun.edu.ng

Phone number: +234-805-550-1032 


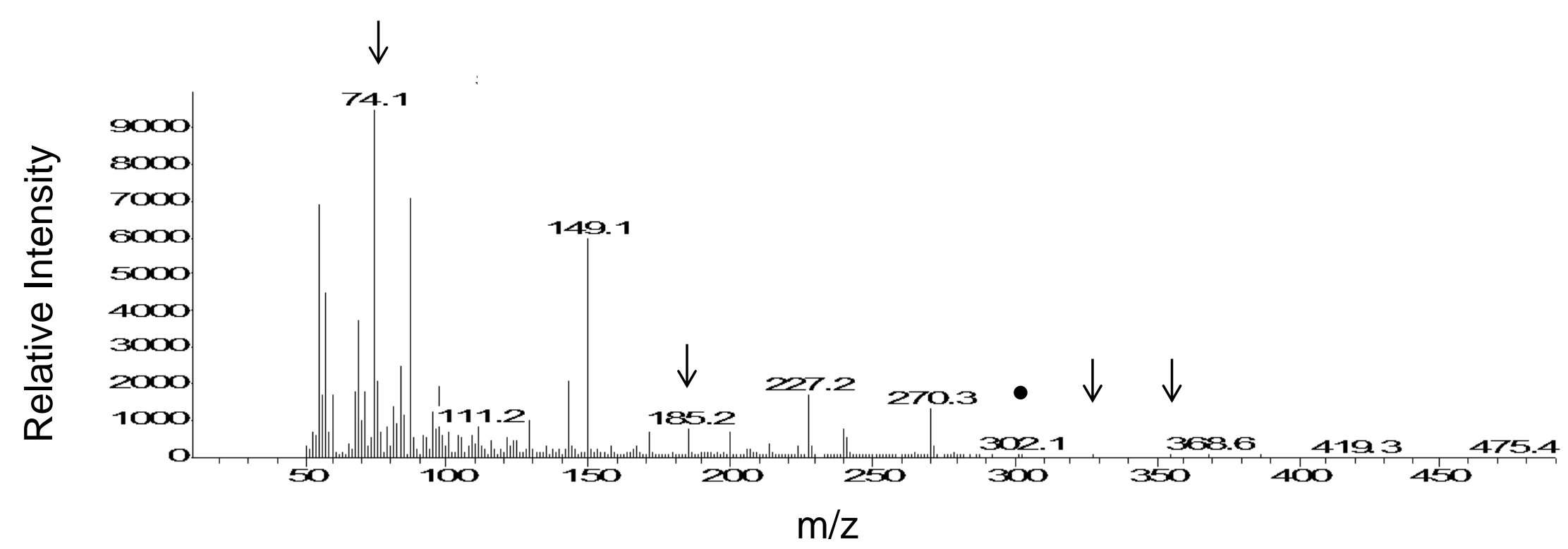

Figure S1. GCMS analysis of lipids in the non-vessel area at embryonic day 1 (ED 1). Retention time=17.403 min. $\mathrm{X}$-axis shows mass to charge $(\mathrm{m} / \mathrm{z})$ and $\mathrm{Y}$-axis shows relative intensity of the ion.

Ion fragments show that increased levels of docosahexaenoic acid (DHA, arrow; 74.1, 185.2, 328, 354) and eicosapentaenoic acid (EPA, dot; 302.1) exist in the nonvessel area. 


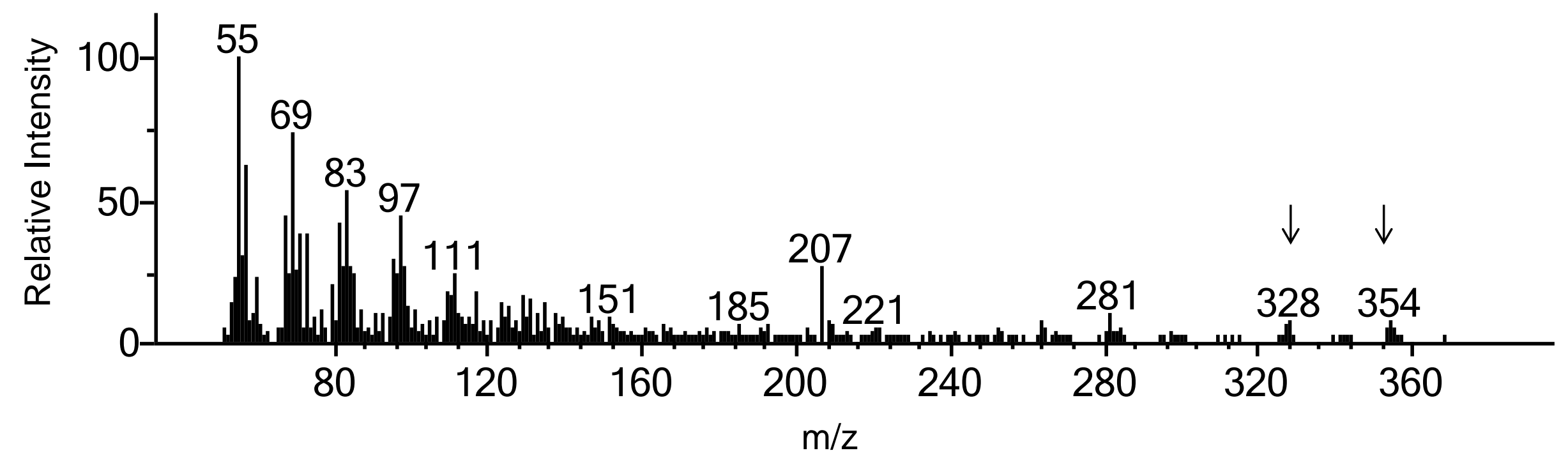

Figure S2. GCMS analysis of lipids in the non-vessel area at embryonic day 3 (ED 3). Retention time=12.702 min. $\mathrm{X}$-axis shows mass to charge $(\mathrm{m} / \mathrm{z})$ and $\mathrm{Y}$-axis shows relative intensity of the ion.

Ion fragments show that high concentration of docosahexaenoic acid (DHA; 328, 354) exists in the non-vessel area. 


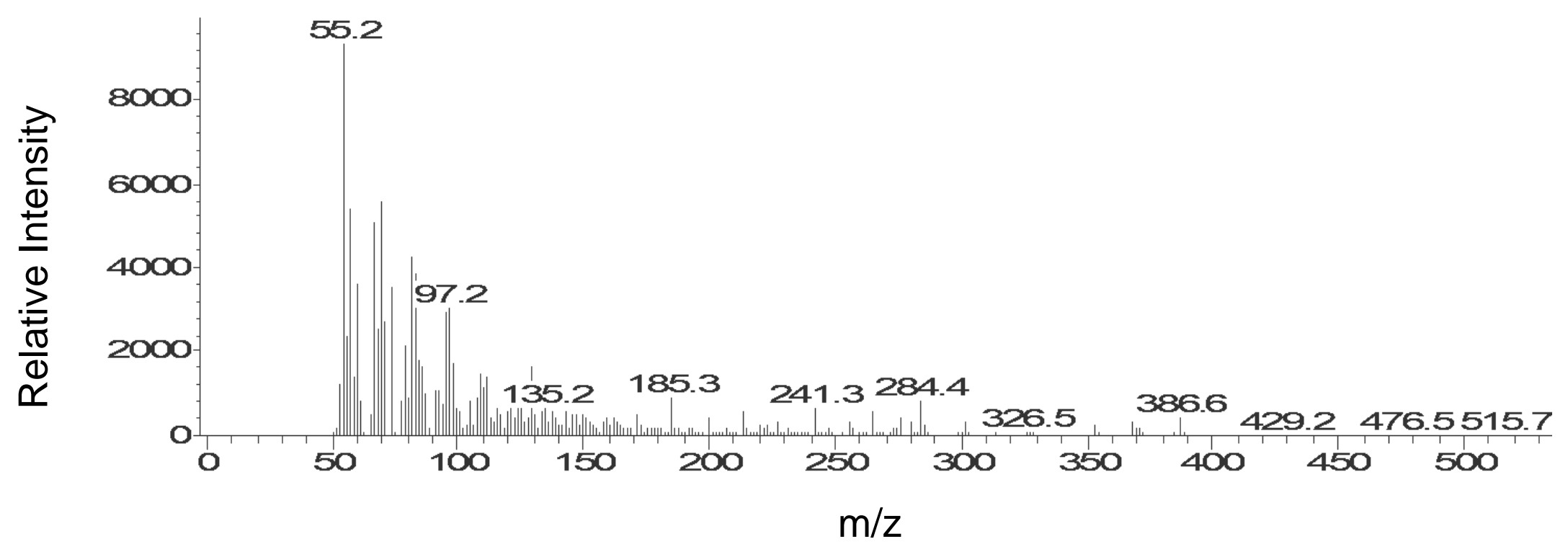

Figure S3. GCMS analysis of lipids in the vessel area at embryonic day 1 (ED 1). Retention time=25.242 min. $\mathrm{X}$-axis shows mass to charge $(\mathrm{m} / \mathrm{z})$ and $\mathrm{Y}$-axis shows relative intensity of the ion.

Ion fragments show that increased levels of 97.2=nitrooleic acid, 135.2=linolenic acid, 185.3=linoleic acid, 200=lauric acid, 241.3=linoleic acid, 256=palmitic acid, 264=oleic acid, 276=lysoPC, 280=linoleic acid, 282=oleic acid, 284.4=stearic acid, 301=cholesterol, 304.4=arachidonic acid, 326.5=nitrooleic acid, 386.6=cholesterol, 429.2=lysophosphatidylcholine (LPC), 476.5=lysophosphatidylethanolamine (LPE, 18:3), and 515.7=lysophosphatidylcholine (LPC, 18:4) exist in the vessel area. 


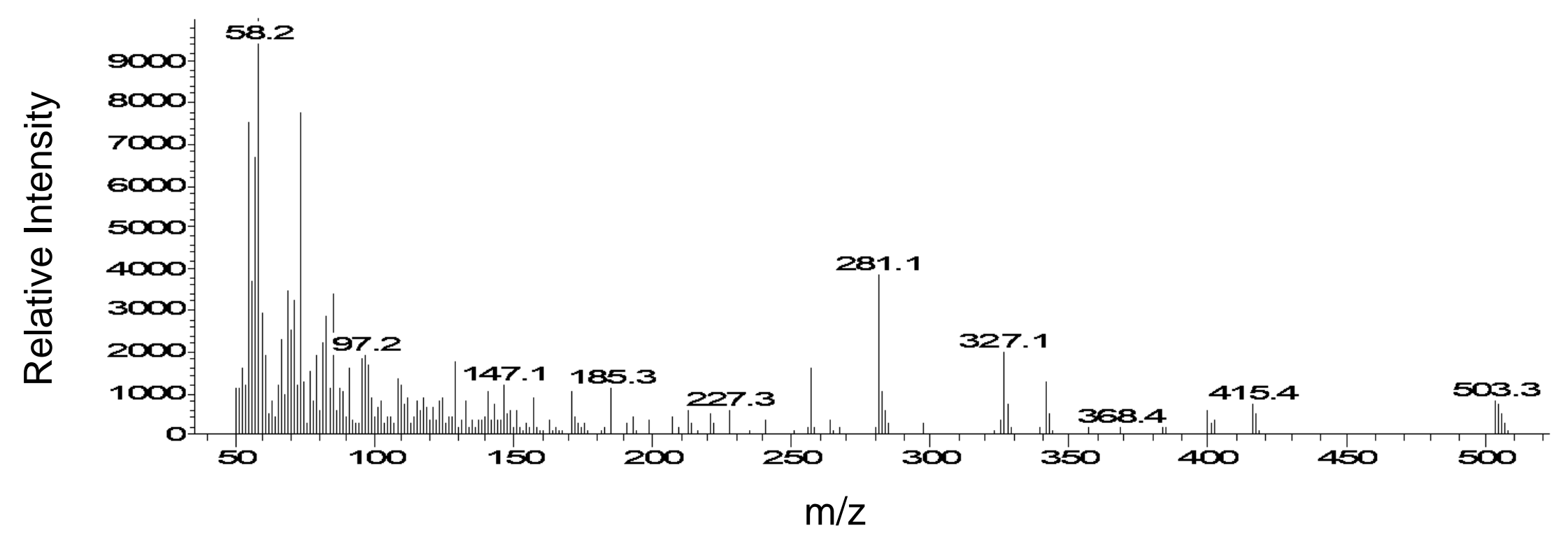

Figure S4. GCMS analysis of lipids in the vessel area at embryonic day 5 (ED 5). Retention time=10.245 min. $\mathrm{X}$-axis shows mass to charge $(\mathrm{m} / \mathrm{z})$ and $\mathrm{Y}$-axis shows relative intensity of the ion.

Ion fragments show that increased levels of oleic acid (97.2), linoleic acid (85.3), palmitic/stearic acid (227.3), cholesterol (281.1, 368.4), nitrooctadecenoic acid (327.1), and lysophosphatidylethanolamine (LPE, 20:3, 503.3) exist in the vessel area. 


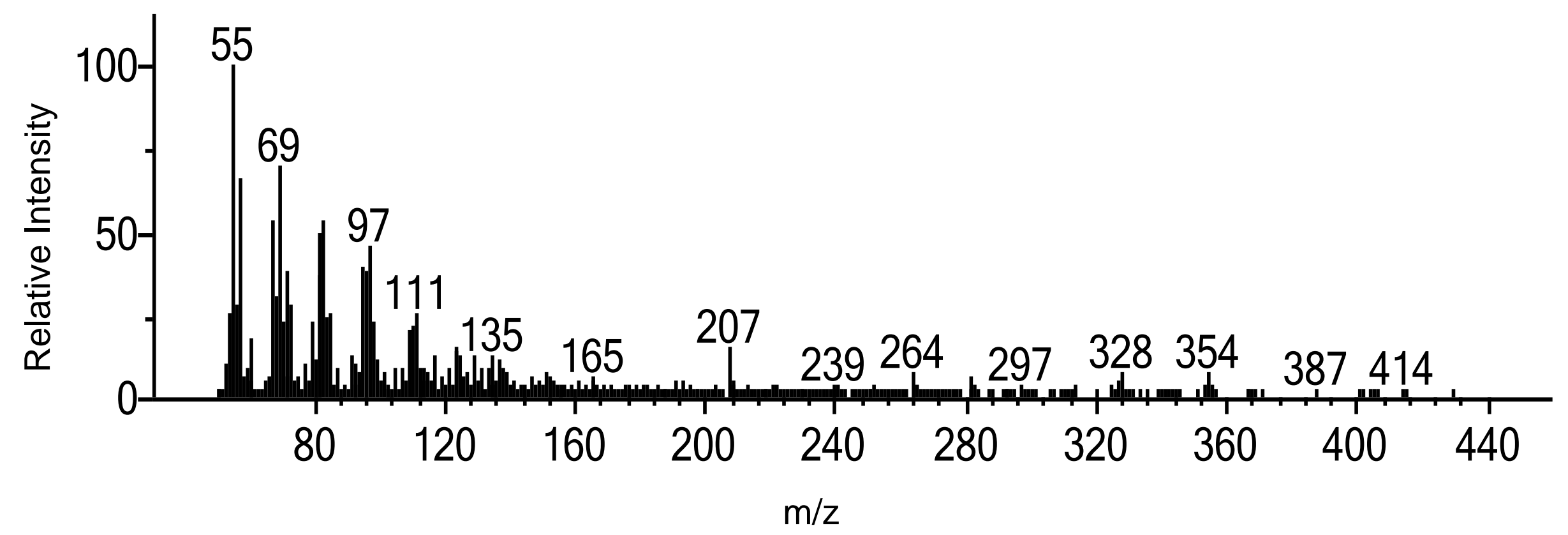

Figure S5. GCMS analysis of lipids in the vessel area at embryonic day 5 (ED 5). Retention time=13.857 min. $\mathrm{X}$-axis shows mass to charge $(\mathrm{m} / \mathrm{z})$ and $\mathrm{Y}$-axis shows relative intensity of the ion.

Ion fragments show that increased levels of oleic acid (111, 165, 207, 264), linolenic acid (135), palmitic acid (239), nitrooleic acid (297), cholesterol (387), 7-ketocholesterol (401), docosahexaenoyllysophosphatidylcholine (DHA-LPC, 414) exist in the vessel area. 


\section{Cholesterol Standard}

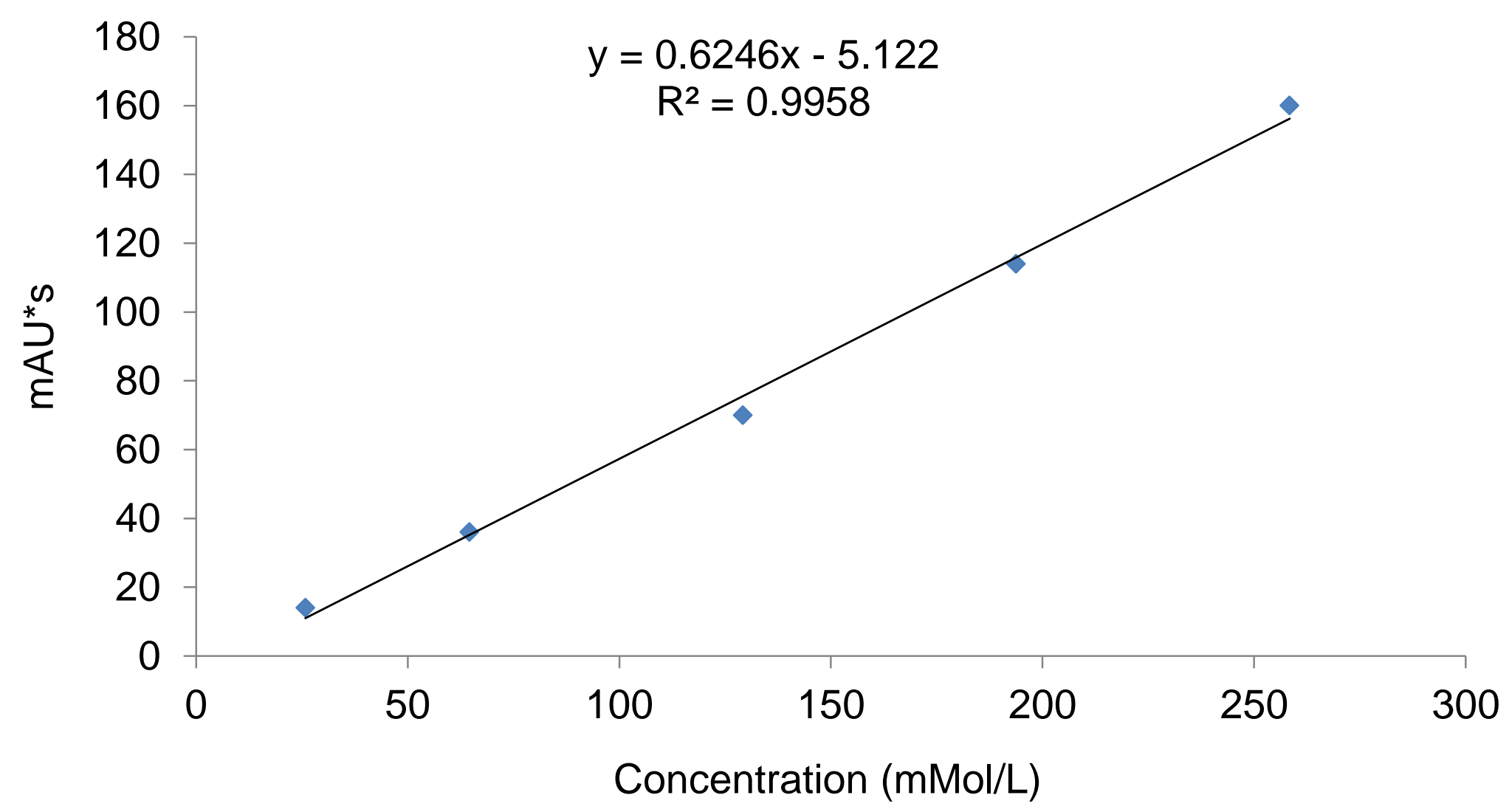

Figure S6. Cholesterol concentration in the chick embryo was determined using the standard curve by quantitative GCMS analysis. The known concentration of cholesterol (X-axis) was injected to GCMS to obtain mAU (Y-axis) value as the relative intensities. Cholesterol levels in the vessel area and nonvessel area were calculated based on the standard curve. The vessel area contains $200 \mathrm{mg}$ of cholesterol whereas $50 \mathrm{mg}$ of cholesterol was measured in the $\mathrm{S} 7$ nonvessel area $(33.4 \%: 8 \%=4.1: 1, \mathrm{p}<0.05, \mathrm{n}=5)$. The same method was applied to calculate oleic acid to demonstrate that the vessel area contains $1.7 \mathrm{~g}$ of oleic acid whereas $309 \mathrm{mg}$ of oleic acid exists in the nonvessel area $(44 \%: 8 \%=5.5: 1, \mathrm{p}<0.05, \mathrm{n}=5)$. 